\title{
Simple Method for Calculating the Weak-Field Electron Diamagnetism in Cubic Lattices
}

\author{
S. OlszeWski \\ Institute of Physical Chemistry of the Polish Academy of Sciences \\ Kasprzaka 44/52, 01-224 Warsaw, Poland
}

(Received August 13, 2001; revised version November 12, 2001)

\begin{abstract}
The idea of electron wave packets moving along closed anisotropic Fermi surfaces placed in a constant magnetic field has been applied to the calculation of the orbital magnetic moment of an individual free electron and tightly-bound $s$-electron in a crystal lattice. In each case the magnetic moment is obtained as a derivative of the electron energy done with respect to the strength of the magnetic field. In the next step, calculations have been extended to electron ensembles. For a free-electron ensemble a thorough calculation of the weak-field magnetic moment gives a result similar to that obtained by a well-known method developed by Landau. On the other hand, calculations done for the $s$-band of the tightly-bound electrons of metallic iron give an absolute value of the magnetic moment much smaller than in the free-electron case. Simultaneously, the sign of the moment is changed indicating a lowering of the iron band electron energy with magnetization.
\end{abstract}

PACS numbers: 75.20.-g, 75.20.En, 76.40.+b

\section{Introduction}

Difficulties connected with the calculation of the diamagnetic susceptibility of the metal electrons are well known [1-6]. When electrons are considered as classical particles, we have a theorem due to Van Leeuwen [5] which states that the orbital magnetic moment vanishes, even if the electrons move in a field which varies from point to point. The non-vanishing diamagnetism of the electron gas is, in fact, explained by quantum mechanics according to which the free-electron 
particles moving in the presence of the magnetic field $\boldsymbol{B}$ occupy discrete levels having the following energies:

$$
E_{n}=\hbar \Omega_{0}\left(n+\frac{1}{2}\right)
$$

here

$$
\Omega_{0}=\frac{e|\boldsymbol{B}|}{m c}
$$

is the gyration frequency of the electron particle in an external magnetic field of the strength $|\boldsymbol{B}|$, the integer number $n$ labels the quantum levels.

When $|\boldsymbol{B}|$ increases, we have an increase in any $E_{n}$ because of relations (1.1) and (1.2). But, simultaneously, an increase in the degeneracy of the quantum levels takes place with the increase in $|\boldsymbol{B}|$. This degeneracy increase provides the system with an empty place in the originally occupied quantum levels, and this place should be next occupied by electrons being originally in the levels of a higher energy. A transition of a part of electrons from the levels of a higher energy into those of a lower energy gives a tendency of lowering the whole energy of the system. In total, an increase in $|\boldsymbol{B}|$ leads to a competition of two tendencies concerning the system energy: the first one - connected with (1.1) and (1.2) - increases that energy, the second one, connected with an increase in degeneracy of any level $n$, tends to make the system energy lower. This situation, which is evidently different from a classical invariance of the electron energy with the change of the field strength $|\boldsymbol{B}|$, led many authors, beginning with Landau, to apply the quantum statistics to the whole of the electron ensemble in order to calculate a net dependence of the free energy of the electron ensemble on the field strength $|\boldsymbol{B}|$.

However, the task successfully accomplished for free electrons, becomes especially tedious in the case of these metals in which the electron particles remain under an important influence of the crystal core [6]. In this case, contrary to the free-electron case, any description of the electron states becomes usually a complicated function of components of the wave vector $k$. In consequence of that, any calculation of the statistical partition function and free energy of the electron ensemble becomes a difficult problem. In fact, the calculation of the Landau levels alone becomes a difficult task in the case of the presence of the crystal lattice, for - already in a semi-classical picture - it requires a regularly complicated calculation of the Roth phase describing the quantum levels. Difficulties with a strictly quantum-mechanical treatment of the crystal electrons submitted to the action of an external magnetic field are well known [7-11]. Simultaneously, an interest in the electron motion of this kind considered especially for simple cubic lattices, has been raised recently on the experimental basis [12-15].

The aim of the present paper is to develop a formalism according to which a treatment of diamagnetism of the crystal electrons seems to be much easier than in the former theories. The approach is based on the observation that although a classical electron energy remains unchanged with the change of the magnetic 
field $|\boldsymbol{B}|$, a quantum energy of that electron is changed because the radius of the electron orbit changes with $|\boldsymbol{B}|$. In effect, a differentiation of the radius of the quantum orbit with respect to the magnetic field leads to a proper expression for the magnetic moment of a single electron. A correct result obtained in this way for an individual magnetic moment can be next integrated to give the magnetic moment of the electron ensemble, both in the case of isotropic and anisotropic (but closed) Fermi surfaces.

\section{Dynamical parameters of electrons in cubic lattices applied to the calculation of the action function and the orbital magnetic moment}

For the sake of simplicity we assume that the field $|\boldsymbol{B}|$ is taken parallelly to one of the Cartesian axis, say $z$, and the same field is also parallel to one of the crystallographic axis. A fundamental parameter of our calculation is the action function

$$
\begin{aligned}
J= & \frac{1}{2 \pi} \oint p \mathrm{~d}\left(\boldsymbol{r}-\boldsymbol{r}_{\mathrm{c}}\right)=\frac{1}{2 \pi} \oint\left[p_{x} \mathrm{~d}\left(x-x_{\mathrm{c}}\right)+p_{y} \mathrm{~d}\left(y-y_{\mathrm{c}}\right)\right] \\
= & \frac{1}{2 \pi} \int_{0}^{T}\left(p_{x} \dot{x}+p_{y} \dot{y}\right) \mathrm{d} t
\end{aligned}
$$

taken along a closed electron path; here $\boldsymbol{r}_{\mathrm{C}}=\left(x_{\mathrm{c}}, y_{\mathrm{c}}\right)$ is the position of the gyration center of the wave packet and the integration in the last step in (2.1) is extended over the time period $T$ of the electron gyration. Since the coordinates of the gyration center are at rest, the velocities of the electron wave packet entering (2.1) can be calculated from the components of the Lorentz equation [16]

$$
\begin{aligned}
& \dot{x}-\dot{x}_{c}=\dot{x} \equiv v_{x}=\frac{\hbar \dot{k}_{y}}{2 \alpha}, \\
& \dot{y}-\dot{y}_{\mathrm{c}}=\dot{y} \equiv v_{y}=-\frac{\hbar \dot{k}_{x}}{2 \alpha},
\end{aligned}
$$

whereas the wave-packet momenta $p_{x}$ and $p_{y}$ are:

$$
\begin{aligned}
& p_{x}=\frac{1}{2} \hbar k_{x}, \\
& p_{y}=\frac{1}{2} \hbar k_{y} .
\end{aligned}
$$

The last two equations can be obtained from the energy formula for a free-electron wave packet moving in the $(x, y)$ plane

$$
E_{\perp}^{\mathrm{free}}=\frac{1}{2} k_{x_{0}}^{2}+\frac{1}{2} k_{y_{0}}^{2}=\frac{1}{2}\left(p_{x}-\alpha y\right)_{0}^{2}+\frac{1}{2}\left(p_{y}+\alpha x\right)_{0}^{2}
$$

the subscript zero refers to free electrons,

$$
\alpha=\frac{e|\boldsymbol{B}|}{2 c}=\frac{e B_{z}}{2 c}
$$


is a constant, moreover in (2.4)

$$
\hbar=m=1
$$

have been put for the sake of simplicity. A reference between $k_{x}, k_{y}, p_{x}, p_{y}$, and $x, y$ in a crystal lattice is discussed in Appendix. From one pair of the Hamilton equations we obtain

$$
\begin{aligned}
& \dot{p}_{x_{0}}=-\frac{\partial E_{\perp}^{\text {free }}}{\partial x_{0}}=-\alpha\left(p_{y}+\alpha x\right)_{0}=-\alpha \dot{y}_{0}=-\alpha k_{y_{0}}, \\
& \dot{p}_{y_{0}}=-\frac{\partial E_{\perp}^{\text {free }}}{\partial y_{0}}=\alpha\left(p_{x}-\alpha y\right)_{0}=\alpha \dot{x}_{0}=\alpha k_{x_{0}},
\end{aligned}
$$

because from the other pair of the Hamilton equations:

$$
\begin{aligned}
& \dot{x}_{0}=v_{x_{0}}=\frac{\partial E_{\perp}^{\mathrm{free}}}{\partial p_{x_{0}}}=\left(p_{x}-\alpha y\right)_{0}=k_{x_{0}} \\
& \dot{y}_{0}=v_{y_{0}}=\frac{\partial E_{\perp}^{\text {free }}}{\partial p_{y_{0}}}=\left(p_{y}+\alpha x\right)_{0}=k_{y_{0}} .
\end{aligned}
$$

The time integration performed in (2.7), (2.7a) gives

$$
\begin{aligned}
& p_{x_{0}}=-\alpha y_{0}+C^{\prime}, \\
& p_{y_{0}}=\alpha x_{0}+C^{\prime \prime},
\end{aligned}
$$

where $C^{\prime}, C^{\prime \prime}$ are constants. But for any rotational motion the integration constants $C^{\prime}, C^{\prime \prime}$ should be necessarily equal to zero. A substitution of (2.9), (2.9a) together with $C^{\prime}=C^{\prime \prime}=0$ into the definitions of $k_{x_{0}}$ and $k_{y_{0}}$ presented in (2.4) [see also (2.8), (2.8a)] provide us readily with the formulae

$$
\begin{aligned}
& p_{x_{0}}=\frac{1}{2} k_{x_{0}}, \\
& p_{y_{0}}=\frac{1}{2} k_{y_{0}},
\end{aligned}
$$

which are equivalent to $(2.3),(2.3 \mathrm{a})$, on condition the simplification done in $(2.6)$ is taken into account.

A substitution of $(2.10),(2.10 \mathrm{a})$ into $(2.7)$, (2.7a) provides us readily with the Lorentz equations; see (2.2), (2.2a). A characteristic point is that the Lorentz equations (2.2), (2.2a), as well as equations (2.3), (2.3a), remain valid not only for a free-electron wave packet but also for a packet moving in the field of a crystal potential. The proof of that property is given in Appendix for the case of the tightly-bound s-electrons in the body-centered cubic lattice; analogous proofs for electrons in other lattices can be also presented.

Since from $(2.2),(2.2 \mathrm{a})$ we have

$$
\frac{\hbar}{2 \alpha} \mathrm{d} k_{x}=-\mathrm{d}\left(y-y_{c}\right)
$$




$$
\frac{\hbar}{2 \alpha} \mathrm{d} k_{y}=\mathrm{d}\left(x-x_{\mathrm{c}}\right)
$$

we obtain for $(2.1)$ on the basis of $(2.3),(2.3 \mathrm{a})$

$$
J=\frac{1}{2 \pi} \frac{\hbar^{2}}{2 \alpha} \oint k_{y} \mathrm{~d} k_{x}=-\frac{1}{2 \pi} \frac{\hbar^{2}}{2 \alpha} \oint k_{x} \mathrm{~d} k_{y} .
$$

The integral expression (2.12) represents the area circumvented by an electron in its gyrational motion performed in the reciprocal space; the coordinates $k_{x}$ and $k_{y}$ can be interchanged on condition the corresponding change of sign before the integral is introduced.

But there exists also another way to represent the integral (2.1)

$$
J=\frac{1}{2 \pi} \oint\left[(-\alpha)\left(y-y_{\mathrm{c}}\right) \mathrm{d}\left(x-x_{\mathrm{c}}\right)+\alpha\left(x-x_{\mathrm{c}}\right) \mathrm{d}\left(y-y_{\mathrm{c}}\right)\right]=\frac{\alpha}{\pi} S
$$

if we note that

$$
S=\frac{1}{2} \oint\left[-\left(y-y_{\mathrm{c}}\right) \mathrm{d}\left(x-x_{\mathrm{c}}\right)+\left(x-x_{\mathrm{c}}\right) \mathrm{d}\left(y-y_{\mathrm{c}}\right)\right]
$$

is the area circumvented by a charged particle gyrating in the ordinary space upon the influence of a constant magnetic field acting along axis $z$. It is evident from (2.13) that $J$ is proportional to $S$ with the proportionality coefficient equal to $\pi^{-1} \alpha$. The ratio between the area circumvented in the reciprocal space and $S$ can be obtained by dividing (2.12) and (2.13)

$$
\frac{\oint k_{y} \mathrm{~d} k_{x}}{S}=\frac{4 \pi \alpha^{2}}{\pi \hbar^{2}}=\left(\frac{e B_{z}}{c \hbar}\right)^{2} .
$$

This is a well-known result (see e.g. [10]) stating that the area circumvented by a particle in an ordinary space can be obtained by a projection of a similar area circumvented in the reciprocal space spanned on the variables $k_{x}$ and $k_{y}$. However, another application of $S$ is that this area, labeled in the free-electron case by $S_{0}$, is proportional to the magnetic moment $M_{z}^{\text {free }}$ produced by a free-electron particle when this particle is moving with a constant velocity

$$
v_{0}=\left(v_{x_{0}}^{2}+v_{y_{0}}^{2}\right)^{1 / 2}
$$

in a plane perpendicular to $B_{z}$. The well-known relation between $M_{z}^{\text {free }}$ and $S_{0}$ is (see e.g. $[17,18]$ )

$$
M_{z}^{\text {free }}=\frac{i}{c} S_{0}
$$

where

$$
i=-\frac{e}{T_{0}}=-\frac{e \Omega_{0}}{2 \pi}
$$

is the electric current calculated for the free-electron gyration period $T=T_{0}$.

When (2.13) is applied to the free-electron action function $J$, labeled henceforth by $J_{0}$, and $S_{0}$, we obtain 


$$
M_{z}^{\text {free }}=\frac{i}{c} S_{0}=-\frac{e \Omega_{0}}{2 \pi c} \frac{\pi J_{0}}{\alpha}=-\frac{e}{m c} J_{0} ;
$$

in (2.14b) we took into account the definition of $\alpha$ given in (2.5) and the formula (1.2) for the free-electron gyration frequency in a field $|\boldsymbol{B}|=B_{z}$. Our aim is now to demonstrate that $M_{z}^{\text {free }}$ identical to $(2.14 \mathrm{~b})$ can be obtained from the derivative of the electron energy done with respect to the field strength $B_{z}$, on condition the well-known quantization rule of the electron action function is simultaneously taken into account $[19,20]$

$$
J_{0}=\hbar\left(n+\frac{1}{2}\right)
$$

$n$ is here an integer number. For free electrons the area in (2.12) becomes a circle,

$$
J=J_{0}=\frac{1}{2 \pi} \frac{\hbar^{2}}{2 \alpha}\left(k_{x_{0}}^{2}+k_{y_{0}}^{2}\right) \pi=\frac{\hbar^{2}}{4 \alpha} a_{0}^{2} .
$$

so a substitution of (2.15) into (2.12a) gives the equation

$$
a_{0}^{2}=k_{x_{0}}^{2}+k_{y_{0}}^{2}=\frac{4 \alpha}{\hbar^{2}} J_{0}=\frac{4 \alpha}{\hbar}\left(n+\frac{1}{2}\right)=\frac{2 e B_{z}}{\hbar c}\left(n+\frac{1}{2}\right) .
$$

The corresponding square radius $r_{0}^{2}$ of the electron orbit in the ordinary space, calculated according to the formula $(2.13 \mathrm{~b})$, is

$$
r_{0}^{2}=\left(\frac{\hbar c}{e B_{z}}\right)^{2} a_{0}^{2}=\frac{2 \hbar c}{e B_{z}}\left(n+\frac{1}{2}\right)
$$

Expression (2.16) is proportional solely to $B_{z}$ (and the constants of nature), so its differentiation gives

$$
\frac{\partial\left(a_{0}^{2}\right)}{\partial B_{z}}=\frac{2 e}{\hbar c}\left(n+\frac{1}{2}\right)=\frac{2 e}{\hbar^{2} c} J_{0}=\frac{2 e}{\hbar^{2} c} \frac{\hbar^{2}}{4 \alpha} a_{0}^{2}=\frac{a_{0}^{2}}{B_{z}} .
$$

We find that the change of the electron amplitude $a_{0}$ in the reciprocal space is a positive number $\left(B_{z}>0\right)$. With the aid of (2.17) the expression (2.16a) gives

$$
\begin{aligned}
\frac{\partial r_{0}^{2}}{\partial B_{z}} & =\left(\frac{\hbar c}{e B_{z}}\right)^{2}\left(-\frac{2}{B_{z}}\right) a_{0}^{2}+\left(\frac{\hbar c}{e B_{z}}\right)^{2} \frac{a_{0}^{2}}{B_{z}}=-\left(\frac{\hbar c}{e B_{z}}\right)^{2} \frac{a_{0}^{2}}{B_{z}} \\
& =-\frac{r_{0}^{2}}{B_{z}}<0,
\end{aligned}
$$

which represents a well-known effect of decrease in the radius $r_{0}$ with an increase in $B_{z}$.

An advantage of the result obtained in (2.17) is that this formula provides us with an expression for the $B_{z}$-derivative of $a_{0}^{2}$ which does not contain any explicit dependence on the quantum level index $n$ : although $a_{0}^{2}$ itself depends on $n$ in the way indicated in (2.16), this dependence does not matter much for the relation (2.17) which remains the same for all $n$. This is a convenient situation because numerous physical parameters, especially the density of electron states, 
can be expressed in terms of $a_{0}$. In consequence, a dependence of the electron observables on $a_{0}$ makes possible for us to integrate the considered observables, for example the magnetic moment, over ensembles of the electron states without making any reference to the quantum number $n$.

An example showing the advantage of the formula (2.17) is its application to the calculation of the magnetic moment $M_{z}^{\text {free }}$ of an individual free-electron particle. Beginning from the energy expression of a single free electron in the plane $\left(k_{x_{0}}, k_{y_{0}}\right)$ [see $\left.(2.16)\right]$, we have

$$
E_{\perp}^{\text {free }}=\frac{\hbar^{2}}{2 m}\left(k_{x_{0}}^{2}+k_{y_{0}}^{2}\right)=\frac{\hbar^{2}}{2 m} a_{0}^{2} \text {; }
$$

the electron mass symbol $m$ and the Planck constant $\hbar$ are recovered. From a definition of the magnetic moment as a derivative of the electron energy done with respect to the field $B_{z}$ we obtain from (2.17) and (2.18)

$$
\begin{aligned}
M_{z}^{\text {free }} & =-\frac{\partial E_{\perp}^{\text {free }}}{\partial B_{z}}=-\frac{\hbar^{2}}{2 m} \frac{\partial}{\partial B_{z}}\left(a_{0}^{2}\right)=-\frac{\hbar^{2}}{2 m} \frac{a_{0}^{2}}{B_{z}}=-\frac{e}{m c} \frac{\hbar^{2}}{4 \alpha} a_{0}^{2} \\
& =-\frac{e}{m c} J_{0} .
\end{aligned}
$$

This is exactly the free-electron magnetic moment $(2.14 \mathrm{~b})$ now obtained from the derivative of the electron energy with respect to $B_{z}$ : the electron energy is changed because the amplitude $a_{0}$ of the electron orbit is changed with the field strength $B_{z}$. For the magnetic moment of the free-electron ensemble we obtain

$$
M_{z}^{\text {gas }}=-V \frac{e^{2}}{m c^{2}} B_{z} k_{\mathrm{F}} \frac{2}{15 \pi}\left(\frac{3}{8 \pi}\right)^{2 / 3}=-0.01029 V \frac{e^{2}}{m c^{2}} B_{z} k_{\mathrm{F}} .
$$

The result (2.19) is quite close to that obtained on the basis of the Fermi-Dirac statistics applied to the Landau levels with the neglected spin degeneracy [5]

$$
M_{Z}^{\text {gas }}=-\frac{1}{3(2 \pi)^{2}} V \frac{e^{2}}{m c^{2}} B_{z} k_{\mathrm{F}}=-0.00844 V \frac{e^{2}}{m c^{2}} B_{z} k_{\mathrm{F}},
$$

for expression (2.19) is different solely by about $15 \%$ from the result given in $(2.20)$. The difference can be attributed to the fact that statistics of the quantum states used in the calculation of (2.19) is that taken from before an application of the external field $B_{z}$.

\section{Orbital magnetic moment of a tightly-bound s-electron}

The orbital magnetic moment of an electron bound in a crystal lattice can be defined as

$$
M_{z}^{\mathrm{latt}}=-\frac{\partial E^{\mathrm{latt}}}{\partial B_{z}}
$$

$E^{\text {latt }}$ is the electron energy. Taking into account the $s$-states forming a band in a cubic lattice we have 


$$
E^{\mathrm{sc}}=E^{(0)}-\alpha-2 \beta \cos \left(k_{x} a_{\text {latt }}\right)+\cos \left(k_{y} a_{\text {latt }}\right)+\cos \left(k_{z} a_{\text {latt }}\right)
$$

for a simple cubic (sc) lattice having the lattice constant $a_{\text {latt }}$,

$$
E^{\mathrm{bcc}}=E^{(0)}-\alpha-8 \beta \cos \left(k_{x} a_{\mathrm{latt}}\right) \cos \left(k_{y} a_{\mathrm{latt}}\right) \cos \left(k_{z} a_{\text {latt }}\right)
$$

for a body-centered cubic (bcc) lattice defined by primitive translations $\boldsymbol{a}_{1}=a_{\text {latt }}(1,1,-1), \boldsymbol{a}_{2}=a_{\text {latt }}(1,-1,1), \boldsymbol{a}_{3}=a_{\text {latt }}(-1,1,1)$,

$$
\begin{aligned}
E^{\mathrm{fCC}} & =E^{(0)}-\alpha-4 \beta\left[\cos \left(k_{x} a_{\text {latt }}\right) \cos \left(k_{y} a_{\text {latt }}\right)+\cos \left(k_{z} a_{\text {latt }}\right) \cos \left(k_{x} a_{\text {latt }}\right)\right. \\
& \left.+\cos \left(k_{y} a_{\text {latt }}\right) \cos \left(k_{z} a_{\text {latt }}\right)\right]
\end{aligned}
$$

for a face-centered cubic (fcc) lattice defined by primitive translations $a_{1}=a_{\text {latt }}(1,1,0), a_{2}=a_{\text {latt }}(1,0,1), a_{3}=a_{\text {latt }}(0,1,1)$ [21]. The parameters $k_{x}, k_{y}$, and $k_{z}$ are Cartesian components of the wave vector $k$ characteristic of an electron state and the constants $E^{(0)}, \alpha$, and $\beta$ denote, respectively, the eigenenergy of an atomic s-state, and the so-called interaction integrals of the crystal Hamiltonian calculated between the atomic orbitals extended on the same atom (symbol $\alpha$ ), and the atomic orbitals located on the nearest-neighbor atoms (symbol $\beta$ ) [21].

The constant components of $E^{\text {latt }}$ in $(3.2)-(3.2 \mathrm{~b})$ do not contribute into $M_{z}^{\text {latt }}$, so $E^{(0)}$ and $\alpha$ can be neglected. Furthermore, it seems to be convenient to transform $E^{\text {latt }}$ with $\alpha=E^{(0)}=0$ and the constants $2 \beta=\beta^{\text {sc }}, 8 \beta=\beta^{\text {bcc }}$, $4 \beta=\beta^{\mathrm{fcc}}$, for the sc, bcc, and fcc lattice, respectively, into the following expressions:

$$
\begin{aligned}
& C^{\mathrm{sc}}=2+\left(\beta^{\mathrm{sc}}\right)^{-1} E^{\mathrm{sc}}+\cos \left(k_{z} a_{\text {latt }}\right)=2-\cos \left(k_{x} a_{\text {latt }}\right)-\cos \left(k_{y} a_{\text {latt }}\right), \\
& C^{\mathrm{bcc}}=1+\frac{E^{\mathrm{bcc}}}{\beta^{\mathrm{bcc}} \cos \left(k_{z} a_{\text {latt }}\right)}=1-\cos \left(k_{x} a_{\text {latt }}\right) \cos \left(k_{y} a_{\text {latt }}\right), \\
& \frac{1}{2}\left[1+\cos \left(k_{z} a_{\text {latt }}\right)\right] C^{\mathrm{fcc}}=\frac{1}{2}\left[1+2 \cos \left(k_{z} a_{\text {latt }}\right)+\left(\beta^{\mathrm{fcc}}\right)^{-1} E^{\mathrm{fcc}}\right] .
\end{aligned}
$$

A characteristic feature of $C^{\mathrm{sc}}, C^{\mathrm{bcc}}$, and $C^{\mathrm{fcc}}$ is that their power expansions done for small values of parameters $k_{x} a_{\text {latt }}, k_{y} a_{\text {latt }}$, and $k_{z} a_{\text {latt }}$ lead to the same free-electron term

$$
C^{\mathrm{sc}}, C^{\mathrm{bcc}}, C^{\mathrm{fcc}} \cong \frac{1}{2}\left(k_{x}^{2}+k_{y}^{2}\right) a_{\text {latt. }}^{2} .
$$

This is a convenient form because (3.4) put equal to the free-electron energy expression $\left(\hbar^{2} / 2 m_{\text {eff }}\right)\left(k_{x}^{2}+k_{y}^{2}\right)$ provides us with a formula for the effective electron mass $m_{\text {eff }}$. Neglecting the constant terms of 2 and $\cos \left(k_{z} a_{\text {latt }}\right)$ in the first step of (3.3), we obtain

for the sc lattice

$$
m_{\mathrm{eff}}^{\mathrm{sc}}=\left|\beta^{\mathrm{sc}} a_{\text {latt }}^{2}\right|^{-1} \hbar^{2},
$$

for the bcc lattice 


$$
m_{\mathrm{eff}}^{\mathrm{bcc}}=\left|\beta^{\mathrm{bcc}} \cos \left(k_{z} a_{\text {latt }}\right) a_{\text {latt }}^{2}\right|^{-1} \hbar^{2},
$$

and for the fcc lattice

$$
m_{\mathrm{eff}}^{\mathrm{fcc}}=\left|\beta^{\mathrm{fcc}}\left[1+\cos \left(k_{z} a_{\text {latt }}\right)\right]\right|^{-1} \hbar^{2} .
$$

Moreover, if we put

$$
k_{x}^{2}+k_{y}^{2}=a_{0}^{2}
$$

in accordance with (2.16), the energy expressions (3.3)-(3.3b) taken on the electron trajectory which is the cross-section line of the surface of the constant energy $E^{\text {latt }}$ with the plane $k_{z}=$ const can be represented by the same formula [16]

$$
C_{t}^{\mathrm{sc}}=C_{t}^{\mathrm{bcc}}=C_{t}^{\mathrm{fcc}}=1-\cos \left(a_{\text {latt }} a_{0}\right)
$$

here the subscript $t$ refers to the electron trajectory. Let us demonstrate for a special case of the fcc lattice that (3.6) applies to the electron trajectories at any $k_{z}$. Putting $a=k_{x} a_{\text {latt }}$ and $b=k_{y} a_{\text {latt }}$ (see (3.3b)) we have

$$
C^{\mathrm{fcc}}=\frac{1+(2-\cos a-\cos b) \cos \left(k_{z} a_{\text {latt }}\right)-\cos a \cos b}{1+\cos \left(k_{z} a_{\text {latt }}\right)} .
$$

If one of parameters $a, b$ entering (3.6a) attains the value of the amplitude of $a_{0}$ on the trajectory of a constant energy, the other parameter is necessarily zero [16], so

$$
\begin{aligned}
C^{\mathrm{fcc}} & =C_{t}^{\mathrm{fcc}}=\frac{1+\left[2-\cos \left(a_{0} a_{\mathrm{latt}}\right)-1\right] \cos \left(k_{z} a_{\text {latt }}\right)-\cos \left(a_{0} a_{\mathrm{latt}}\right)}{1+\cos \left(k_{z} a_{\text {latt }}\right)} \\
& =1-\cos \left(a_{0} a_{\text {latt }}\right) .
\end{aligned}
$$

This formula, obviously independent of $k_{z}$, is valid for the whole trajectory of a constant energy.

In the presence of the magnetic field the electrons gyrate along their trajectories, so

$$
E^{\text {latt }}=E_{t}^{\text {latt }}
$$

Therefore, when (3.1) is applied to a tightly-bound s-electron in the bcc lattice taken as an example, we obtain

$$
M_{z}^{\mathrm{bcc}}=-\frac{\partial E_{t}^{\mathrm{bcc}}}{\partial B_{z}}=-\frac{\partial}{\partial B_{z}}\left[\beta^{\mathrm{bcc}} \cos \left(k_{z} a_{\mathrm{latt}}\right) C_{t}^{\mathrm{bcc}}\right]
$$

because the derivatives of the constant terms can be neglected. Taking into account (2.17) we obtain

$$
\begin{aligned}
M_{z}^{\mathrm{bcc}} & =-\beta^{\mathrm{bcc}} \cos \left(k_{z} a_{\text {latt }}\right) \frac{\partial C_{t}^{\mathrm{bcc}}}{\partial a_{0}^{2}} \frac{\partial a_{0}^{2}}{\partial B_{z}} \\
& =-\frac{1}{2} \beta^{\mathrm{bcc}} \sin \left(a_{\text {latt }} a_{0}\right) \frac{a_{\text {latt }} a_{0}}{B_{z}} \cos \left(k_{z} a_{\text {latt }}\right) .
\end{aligned}
$$

For a not too large $a_{0}$ we may put $\sin \left(a_{\text {latt }} a_{0}\right) \approx a_{\text {latt }} a_{0}$. A substitution of $k_{z}=0$ done for the sake of simplicity allows us to estimate the ratio 


$$
\frac{M_{z}^{\mathrm{bcc}}}{M_{z}^{\text {free }}} \cong \frac{1}{2} \frac{\beta^{\mathrm{bcc}}\left(a_{\mathrm{latt}} a_{0}\right)^{2}}{B_{z}} \frac{2 m c \alpha}{e \hbar^{2} a_{0}^{2}}=\frac{\beta^{\mathrm{bcc}}\left(a_{\mathrm{latt}}\right)^{2} m}{2 \hbar^{2}}
$$

for a single electron; $M_{z}^{\text {free }}$ is the magnetic moment taken from (2.18a). The $\beta^{\text {bcc }}$ integral, estimated for the $4 s$-electrons in a metallic iron, is approximately equal to $[22]$

$$
\beta^{\mathrm{bcc}} \cong-0.45 \mathrm{Ry},
$$

whereas the experimental $\alpha_{\text {latt }}$ for the bcc iron crystal is equal to $2.86 \times 10^{-8} \mathrm{~cm}[23]$.

The formula (3.8) can be expressed in terms of the Bohr magneton units by dividing it by parameters characteristic of the first Bohr atomic orbit of hydrogen for which we have Ry $a_{\mathrm{B}}^{2}\left(2 m / \hbar^{2}\right)=1 ; a_{\mathrm{B}}$ is the orbit radius. This substitution introduced into (3.8) gives

$$
\frac{\frac{1}{4}\left|\beta^{\mathrm{bcc}}\right|}{\mathrm{Ry}}\left(\frac{a_{\text {latt }}}{a_{\mathrm{B}}}\right)^{2} \cong 3.3 \text {. }
$$

The result of (3.10) shows that the size of the magnetic moment calculated for the tightly-bound Fe atom in a crystal lattice of metallic iron is not much different from the weighted average of the experimental magnetic moment observed for the Fe atom bound in the crystal lattices of metals other than Fe. For example, the average magnetic moment of the atomic Fe measured in various second-row transition metals (from $\mathrm{Nb}$ to $\mathrm{Cd}$ for which we assume that the binding parameters of the Fe atom are not much different than the parameters for the same atom bound in the metallic iron) is equal to about $2.8 \mu_{\mathrm{B}}$ [24].

\section{The weak-field magnetic moment calculated for an ensemble of the tightly-bound $s$-electrons in a metallic iron}

Usually, an approach to the diamagnetic susceptibility of the tightly-bound electrons in metals is classified as a not easy task [6]. A difficulty lies in obtaining, in the first step, the Landau levels for such electrons and next in a statistical treatment which is necessary to be done for ensembles of such levels. Peierls' [2] original proposal of the formulae for the diamagnetic susceptibility of the tightly-bound electrons is in fact limited to a free-electron approach supplemented by the notion of the effective electron mass, as it has been pointed out by Fröhlich [25].

Our approach is based on the lines presented in Secs. 2 and 3. First, we examine the magnetic moment for a slice of a constant $k_{z}$. The change of the electron energy due to a change of $B_{z}$ can be obtained beginning with the states which lie on the circumference of the area defined by $J$. The length of that circumference is represented by the derivative

$$
\frac{\partial J}{\partial a_{0}}=\frac{\partial J}{\partial C_{t}^{\text {latt }}} \frac{\partial C_{t}^{\text {latt }}}{\partial a_{0}}=\frac{1}{\omega^{J}} \frac{\partial C_{t}^{\text {latt }}}{\partial a_{0}} .
$$

In the second step in (4.1) we took into account that [16] 


$$
\omega^{J}=\left(\frac{\partial C_{t}^{\mathrm{latt}}}{\partial J}\right)^{-1}=\omega .
$$

In a special case of free electrons we obtain for the circumference of $J_{0}$

$$
\frac{\partial J_{0}}{\partial a_{0}}=\frac{\hbar^{2}}{4 \pi \alpha} 2 \pi a_{0}=\frac{\hbar^{2}}{2 \alpha} a_{0},
$$

which is in agreement with (4.1) because

$$
\frac{\partial J_{0}}{\partial a_{0}}=\frac{1}{\Omega_{0}} \frac{\partial E_{\perp}^{\text {free }}}{\partial a_{0}}=\frac{m c}{e B_{z}} \frac{\hbar^{2}}{m} a_{0}=\frac{\hbar^{2}}{2 \alpha} a_{0}
$$

see (1.2), (2.5), and (2.18).

Any circumference of $J$ having the length $\partial J / \partial a_{0}$ carries the energy proportional to

$$
C_{t}^{\operatorname{latt}} \frac{\partial J}{\partial a_{0}},
$$

because, by definition, $J$ is a surface area limited by a line of a constant energy equal to $E_{t}^{\text {latt }}$, and $C^{\text {latt }}$ is proportional to $E^{\text {latt }}$ for a constant $k_{z}$; see $(3.3)-(3.3 \mathrm{~b})$. In a plane of $k_{z}=$ const the expression $C_{t}^{\text {latt }}$ is a function of $a_{0}$, therefore a contribution given to the magnetic moment by the energy (4.4) is

$$
\begin{gathered}
M_{z}^{\text {circ latt }}=-\frac{\partial}{\partial B_{z}}\left(C_{t}^{\text {latt }} \frac{\partial J}{\partial a_{0}}\right)=-\frac{\partial}{\partial a_{0}^{2}}\left(C_{t}^{\text {latt }} \frac{\partial J}{\partial a_{0}}\right) \frac{\partial a_{0}^{2}}{\partial B_{z}} \\
=-\frac{\partial}{2 a_{0} \partial a_{0}}\left(C_{t}^{\text {latt }} \frac{\partial J}{\partial a_{0}}\right) \frac{a_{0}^{2}}{B_{z}} .
\end{gathered}
$$

The magnetic moment for a slice of quantum states being in the plane $k_{z}=$ const can be obtained from (3.7a) when: (i) the expression for $M_{z}^{\text {bcc }}$ is multiplied by the electron number present in an elemental area of a slice which for the tightly-bound s-electrons is ( $\mathrm{d} a_{0}$ is an infinitesimal increase in $a_{0}$ )

$$
\frac{4 \pi \alpha}{\hbar^{2}} \frac{\partial J}{\partial a_{0}} \frac{\mathrm{d} a_{0}}{(2 \pi)^{2}}\left(a_{\text {latt }}\right)^{2} ;
$$

(ii) the result of multiplication is integrated in the whole interval of $a_{0}$ of the occupied states in the slice $\left(a_{0}^{\mathrm{F}^{\prime}}\right.$ is the limiting $a_{0}$ for the slice)

$$
0<a_{0}<a_{0}^{\mathrm{F}^{\prime}}
$$

The fact that $a_{\text {latt }}$ in (4.6) refers to the edge of an elementary cube occupied by one electron does not matter because the density of states in an empty lattice is here considered, and in a final result the parameter $a_{\text {latt }}$ entering (4.6) is transformed into that corresponding to the bcc lattice.

In general, the action function

$$
J=J\left(a_{0}\right)
$$


of the tightly-bound electrons which enters (4.6) has a much more complicated dependence on $a_{0}$ than the free-electron $J_{0}$ given in (2.12a). But for $s$-electrons in cubic lattices examined in the present paper the difference

$$
\Delta J=J\left(a_{0}\right)-J_{0}\left(a_{0}\right)
$$

is not a large quantity and in the first approximation can be put equal to zero. In effect, an estimate of the magnetic moment for a slice of the tightly-bound $s$-electrons can be obtained by an integration similar to that performed for an area of the free-electron quantum states; we neglect here also deviations from a circular shape of the electron orbit in the $\left(k_{x}, k_{y}\right)$-space obtained in effect of the presence of the crystal lattice. In consequence, the magnetic moment in a slice of states is

$$
\begin{aligned}
M_{z}^{\text {slice }} & =\frac{1}{2 B_{z}}\left(-\beta^{\text {latt }}\right) 2 \pi \int_{0}^{a_{0}^{\mathrm{F}^{\prime}}} a_{0}^{2} \mathrm{~d} a_{0} a_{\text {latt }} \frac{\left(a_{\text {latt }}\right)^{2}}{(2 \pi)^{2}} \sin \left(a_{0} a_{\text {latt }}\right) \cos \left(k_{z} a_{\text {latt }}\right) \\
= & -\frac{\beta^{\text {latt }}}{4 B_{z} \pi}\left\{2 a_{0}^{\mathrm{F}^{\prime}} a_{\text {latt }} \sin \left(a_{0}^{\mathrm{F}^{\prime}} a_{\text {latt }}\right)-\left[\left(a_{0}^{\mathrm{F}^{\prime}} a_{\text {latt }}\right)^{2}-2\right] \cos \left(a_{0}^{\mathrm{F}^{\prime}} a_{\text {latt }}\right)-2\right\} \\
& \times \cos \left(k_{z} a_{\text {latt }}\right) .
\end{aligned}
$$

This formula can be integrated over the variable $k_{z}$ by making a free-electron substitution for $a_{0}^{\mathrm{F}^{\prime}}$ which is $a_{0}^{\mathrm{F}^{\prime}}=\left(k_{\mathrm{F}}^{2}-k_{z}^{2}\right)^{1 / 2} ; k_{\mathrm{F}}$ is the wave vector at the Fermi level. The density of states along the $k_{z}$-axis is

$$
\mathrm{d} n_{z}=\frac{L}{2 \pi} \mathrm{d} k_{z}
$$

where $L$ is the length of the cylinder representing the metal volume. Taking the interval of the occupied $k_{z}$ equal to $-k_{\mathrm{F}}<k_{z}<k_{\mathrm{F}}$, the integral of (4.10) over $k_{z}$ gives

$$
\begin{gathered}
-\frac{L}{\pi} \frac{\beta^{\text {latt }}}{4 B_{z} \pi} a_{\text {latt }}^{4} k_{\mathrm{F}}^{5}\left[\frac{2}{15}-\frac{1}{45}\left(a_{\text {latt }} k_{\mathrm{F}}\right)^{2}+\frac{79}{56700}\left(a_{\text {latt }} k_{\mathrm{F}}\right)^{4}-\frac{37}{793800}\left(a_{\text {latt }} k_{\mathrm{F}}\right)^{6}\right. \\
\left.+\frac{7963}{81729648 \times 10^{2}}\left(a_{\text {latt }} k_{\mathrm{F}}\right)^{8}-\frac{37649}{2697078384 \times 10^{3}}\left(a_{\text {latt }} k_{\mathrm{F}}\right)^{10}+\ldots\right] \\
=-\frac{L \beta^{\text {latt }}}{4 B_{z} \pi^{2}} a_{\text {latt }}^{4} k_{\mathrm{F}}^{5}(0.13333-0.21268+0.12763-0.04086+0.00817 \\
-0.00112+\ldots)=-\frac{L \beta^{\text {latt }}}{4 B_{z} \pi^{2}} a_{\text {latt }}^{4} k_{\mathrm{F}}^{5} 0.01447
\end{gathered}
$$

on condition the empty-lattice relation

$$
\frac{a_{\text {latt }}}{2 \pi}\left(\frac{8 \pi}{3}\right)^{1 / 3} k_{\mathrm{F}}=1
$$

is assumed as approximately applicable also in the tight-binding (t.b.) case.

The convergence of the series given in (4.11), beginning with the third term of the series, is found to be quite rapid. Basing on relation $(2.13 \mathrm{~b})$ applied to the 
metal volume cylinder having the base $S=\pi R^{2}$, relation (4.11a), and the formula $\pi R^{2} L=V$ for the cylinder volume $V$, expression (4.11) can be transformed into

$$
M_{2}^{\text {t.b. }}=-\beta^{\text {latt }} \frac{V}{\pi} \frac{e^{2} a_{\text {latt }}^{2}}{(\hbar c)^{2}} B_{z} k_{\mathrm{F}}\left(\frac{3}{8 \pi}\right)^{2 / 3} 0.01447 .
$$

The ratio between $M_{z}^{\text {t.b. }}$ calculated in (4.12) and $M_{z}^{\text {gas }}$ calculated in (2.19) becomes

$$
\frac{M_{z}^{\mathrm{t} . \mathrm{b}}}{M_{z}^{\text {gas }}}=\frac{\beta^{\mathrm{latt}} \frac{V}{\pi} \frac{e^{2}}{(\hbar c)^{2}} B_{z} k_{\mathrm{F}} 0.01447 a_{\text {latt }}^{2}}{V \frac{e^{2}}{m c^{2}} B_{z} k_{\mathrm{F}} \frac{2}{15 \pi}}=\frac{\beta^{\mathrm{latt}} m a_{\text {latt }}^{2}}{\hbar^{2}} 0.1085 .
$$

The size of $\beta^{\text {latt }}=\beta^{\text {bcc }}$ has been estimated from a half-width of the band of the tightly-bound $4 s$ states in the bcc iron [22]; see (3.9). A negative sign of $\beta^{\text {latt }}=\beta^{\text {bcc }}$

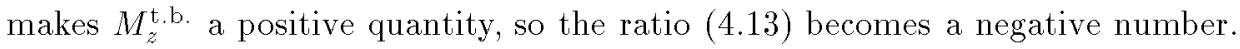
If the volume occupied per one $4 s$ electron of the Fe atom is considered, the edge of the cube representing that volume is

$$
2^{-1 / 3} a_{\mathrm{c}} \cong 2^{-1 / 3} \times 2.86 \times 10^{-8} \mathrm{~cm}=2.27 \times 10^{-8} \mathrm{~cm} ;
$$

here the crystallographic data for the edge $a_{c}$ of the elementary cell in the $\alpha$-Fe lattice are taken into account [23]. Expression (4.14) should replace $a_{\text {latt }}$ entering (4.11)-(4.13). A substitution of (3.9) and (4.14) into (4.13) gives

$$
\left|\frac{M_{z}^{\text {t.b. }}}{M_{z}^{\text {gas }}}\right| \cong 0.033
$$

This ratio is much smaller than a similar ratio $\left|\frac{M_{z}^{\text {bcc }}}{M_{z}^{\text {free }}}\right| \cong 3.3$ calculated in $(3.10)$ for the magnetic moment of a single tightly-bound s-electron in the bcc lattice. A reason of the difference is a strongly oscillating behavior of the expression for $M_{z}^{\text {slice }}$ obtained in (4.10).

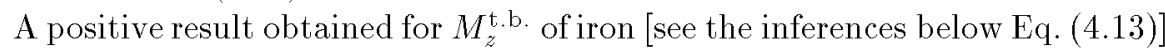
implies a non-vanishing magnetic moment above the Curie point because of the expected only mild influence of temperature on the calculated electronic properties. In fact, the presence of the magnetic moment above the Curie point of the metallic Fe is confirmed long time ago on the empirical basis [26].

\section{Summary}

The idea that the tightly-bound electrons in a crystal lattice submitted to the action of a constant magnetic field $B_{z}$ behave like classical particles has been raised rather recently by Suhl [27]. A detailed solution of the motion equations derived in the Suhl problem, when this problem is extended to all cubic lattices, has been done in [16].

In the present paper the old problem of diamagnetism, applied equally to free electrons and tightly-bound s-electrons moving in a regular crystal lattice, 
has been approached in a much more simple way than that proposed before by many authors. The main simplification is due to the fact that the dependence of the amplitude $a_{0}$ of the free-electron motion in a constant magnetic field on the strength $B_{z}$ of that field can be readily calculated. In the next step, this dependence is applied in calculating the derivative of the free-electron energy with respect to the field $B_{z}$. For a single electron, the accuracy of the orbital magnetic moment obtained in this way is checked by comparing it with the magnetic moment calculated directly from the electric current provided by the gyrating electron particle. A similar comparison of the former and present theory is done for the magnetic moment of an ensemble of the free-electron particles filling the electron gas volume.

Since energy and density number of the tightly-bound $s$-electrons in a crystal lattice can be represented as functions of the same free-electron amplitude $a_{0}$, the calculation of diamagnetism of the tightly-bound electrons can be done in a way which is much similar to that applied for the free-electron case. A further simplification comes from the fact that the electron energies can be referred solely to the states distributed along the electron trajectories in the reciprocal space [16]. This makes the corresponding tight-binding expressions for the electron energy of an especially simple form; see (3.6). The case of $4 s$ electrons moving in the bcc lattice of the metallic iron has been taken as an example of the numerical calculations. Here the interaction integral of the tightly-bound electrons with the atomic core is assumed to be a known parameter. The magnetic moment of an individual tightly-bound $s$-electron (calculated for a not too large $a_{0}$ ), as well as that of an ensemble of the tightly-bound s-electrons, are shown, unlike the moments of the corresponding free electron objects, to be positive numbers. This shows a tendency of lowering the energy of an electron ensemble with an increase in the absolute value of the magnetic field. On the other hand, the absolute value of the magnetic moment of a single tightly-bound electron becomes evidently larger than the analogous moment of a free-electron, but for the electron ensembles an opposite relation between the absolute sizes of the magnetic moments is obtained.

In Appendix we demonstrate a partition of the wave-vector components, $k_{x}$ and $k_{y}$, of a tightly-bound $s$-electron moving in a magnetic field into two equal parts: one of these wave-vector parts depends solely on the electron position, whereas the other part is equal to the electron momentum; here both parameters of position and momentum are considered in the ordinary space. This property of partition of $k_{x}, k_{y}$ (demonstrated for the bcc lattice) leads, in the first step, to the Lorentz equation, in the next step, we obtain the equations referring the wave-vector components to the electron momenta. The same equations which couple $k_{x}, k_{y}$ with coordinates $x, y$ and momenta $p_{x}, p_{y}$ in the bec lattice can be readily obtained for the tightly-bound s-electrons in the sc lattice, the fcc lattice, as well as in the free-electron case; see Sec. 2. 


\section{Appendix}

Our aim is to demonstrate that components of the wave-vector parameters

$$
\begin{aligned}
& \hbar k_{x}=p_{x}+\frac{e}{c} A_{x}=p_{x}-\alpha y, \\
& \hbar k_{y}=p_{y}+\frac{e}{c} A_{y}=p_{y}+\alpha x,
\end{aligned}
$$

which characterize the motion of the tightly-bound $s$-electron wave packets in the plane $(x, y)$ being perpendicular to the magnetic field $B_{z}$, satisfy the equations

$$
\begin{aligned}
& \frac{1}{2} \hbar k_{x}=p_{x}=-\alpha y, \\
& \frac{1}{2} \hbar k_{y}=p_{y}=\alpha x,
\end{aligned}
$$

$\alpha$ is a constant given in (2.5), $A_{x}=-\frac{1}{2} B_{z} y, A_{y}=\frac{1}{2} B_{z} x$ are components of the vector potential taken in a circular gauge; see e.g. [10]. For the case of free electrons equations (A.2) and (A.2a) are derived in Sec. 2, for the tightly-bound s-electrons the derivation given below is limited to the case of the bcc lattice; a treatment of the tightly-bound electrons in the sc and fcc lattices is exactly the same. For the sake of simplicity we put henceforth $a_{\text {latt }}=1$.

A convenient Hamiltonian expression for electrons in the bcc lattice becomes that represented by $(3.3 \mathrm{a})$

$$
C^{\mathrm{bcc}}=1-\cos a \cos b
$$

where we applied the abbreviations

$$
\begin{aligned}
& a=k_{x}=p_{x}-\alpha y, \\
& b=k_{y}=p_{y}+\alpha x .
\end{aligned}
$$

From (A.3), (A.4), and (A.4a) we derive the Hamilton equations

$$
\begin{aligned}
& \dot{x}=\frac{\partial C^{\mathrm{bcc}}}{\partial p_{x}}=\sin a \cos b, \\
& \dot{y}=\frac{\partial C^{\mathrm{bcc}}}{\partial p_{y}}=\cos a \sin b, \\
& \dot{p}_{x}=-\frac{\partial C^{\mathrm{bcc}}}{\partial x}=-\alpha \cos a \sin b=-\alpha \dot{y}, \\
& \dot{p}_{y}=-\frac{\partial C^{\mathrm{bcc}}}{\partial y}=\alpha \sin a \cos b=\alpha \dot{x} .
\end{aligned}
$$

From (A.4), (A.4a) and (A.5)-(A.8) we obtain

$$
\begin{aligned}
& \dot{a}=\dot{p}_{x}-\alpha \dot{y}=2 \dot{p}_{x}=-2 \alpha \dot{y}=-2 \alpha \cos a \sin b, \\
& \dot{b}=\dot{p}_{y}+\alpha \dot{x}=2 \dot{p}_{y}=2 \alpha \dot{x}=2 \alpha \sin a \cos b
\end{aligned}
$$


The second time differentiation of (A.9) gives

$$
\ddot{a}=-2 \alpha(\dot{a} \sin a \sin b+\dot{b} \cos a \cos b)=-2 \alpha^{2} \sin 2 a,
$$

and precisely the same formula can be obtained from (A.9a) for the variable $b$. The formula (A.10) can be transformed into inhomogeneous differential equation for a harmonic oscillator

$$
\ddot{a}+4 \alpha^{2} a=-4 \alpha^{2}(\cos a \sin a-a),
$$

which for a small $a$ (and a small amplitude $a_{0}$ ) tends to the harmonic oscillator equation with a frequency $\Omega_{0}^{\text {bcc }}=2 \alpha$. Because of (2.5) we have $\Omega_{0}^{\text {bcc }}$ proportional to $B_{z}$.

Similar calculations done for the tightly-bound s-electrons in the sc and fcc lattices give also

$$
\begin{aligned}
& \dot{a}=\dot{p}_{x}-\alpha \dot{y}=2 \dot{p}_{x}=-2 \alpha \dot{y}, \\
& \dot{b}=\dot{p}_{y}+\alpha \dot{x}=2 \dot{p}_{y}=2 \alpha \dot{x},
\end{aligned}
$$

which are equations (A.9) and (A.9a) obtained for the bcc lattice. The integration of (A.12), (A.12a) can be performed in the same way as it was applied for free electrons [see (2.9) and (2.9a)] giving the result presented in (A.2), (A.2a). The frequencies of electron gyration obtained for a small $a_{0}$ are

$$
\Omega_{0}^{\mathrm{sc}}=2 \alpha\left(1-C^{\mathrm{sc}}\right)^{1 / 2}
$$

and

$$
\Omega_{0}^{\mathrm{fcc}}=\frac{2 \alpha}{\left(1+\cos k_{z}\right)^{1 / 2}}\left(1+\cos k_{z}-C^{\mathrm{fcc}} \cos k_{z}\right)^{1 / 2},
$$

which is a $k_{z}$-dependent frequency. The electron mass which should be substituted in calculating the effective $\Omega_{0}^{\text {latt }}$ is that given in (3.5)-(3.5b). A solution of equations of the kind given in (A.11) can be done for any cubic lattice along the lines outlined in [16].

\section{References}

[1] L. Landau, Z. Phys. 64, 629 (1930).

[2] R. Peierls, Z. Phys. 80, 783 (1933); ibid. 81, 186 (1933)

[3] R. Peierls, Quantum Theory of Solids, Oxford University Press, New York 1955.

[4] A.H. Wilson, Proc. Camb. Philos. Soc. 49, 292 (1953).

[5] N.F. Mott, H. Jones, The Theory of the Properties of Metals and Alloys, University Press, Oxford 1936

[6] J. Callaway, Quantum Theory of the Solid State, 2nd ed., Academic, New York 1991.

[7] P.G. Harper, Proc. Phys. Soc. (London) A 68, 874 (1955).

[8] D.R. Hofstadter, Phys. Rev. B 14, 2239 (1976). 
[9] G.H. Wannier, Revs. Mod. Phys. 34, 645 (1962).

[10] J.C. Slater, Quantum Theory of Molecules and Solids, Vol. 3, McGraw-Hill, New York 1967.

[11] J.M. Ziman, Principles of the Theory of Solids, University Press, Cambridge 1972.

[12] S.J. Blundell, J. Singleton, Phys. Rev. B 53, 5609 (1996).

[13] J. Singleton, F.L. Pratt, M. Doporto, T.J.B.M. Janssen, M. Kurmoo, J.A.A.J. Perenboom, W. Hayes, P. Day, Phys. Rev. Lett. 68, 2500 (1992).

[14] S.J. Blundell, A. Ardavan, J. Singleton, Phys. Rev. B 55, R6129 (1997).

[15] A. Ardavan, J.M. Schrama, S.J. Blundell, J. Singleton, W. Hayes, M. Kurmoo, P. Day, P. Goy, Phys. Rev. Lett. 81, 713 (1998).

[16] S. Olszewski, T. Roliński, T. Kwiatkowski, Phys. Rev. B 59, 3740 (1999).

[17] H. Goldstein, Classical Mechanics, 2nd. ed., Addison-Wesley, Reading (Massachusetts) 1980.

[18] W. Greiner, Classical Electrodynamics, Springer, New York 1998.

[19] L. Onsager, Philos. Mag. 43, 1006 (1952).

[20] C. Kittel, Introduction to Solid State Physics, 6th ed., Wiley, New York 1996.

[21] J.R. Reitz, in: Solid State Physics, Eds. F. Seitz, D. Turnbull, Vol. 1, Academic, New York 1955, p. 1.

[22] F. Stern, Phys. Rev. 116, 1399 (1959).

[23] B.F. Ormont, Structures of Inorganic Materials, GITTL, Moscow 1950 (in Russian).

[24] A.M. Clogston, B.T. Matthias, M. Peter, H.J. Williams, E. Corenzwit, R.C. Sherwood, Phys. Rev. 125, 541 (1962).

[25] H. Fröhlich, Elektronentheorie der Metalle, Springer, Berlin 1936.

[26] R. Gersdorf, in: Metallic Solid Solutions, Eds. J. Friedel, A. Guinier, Benjamin, New York 1963, p. XXVI-1.

[27] H. Suhl, J. Phys. (Paris) 50, 2613 (1989). 